\title{
SemOutQuote: Using Semantics for Innovation Offshore Outsourcing Management
}

\author{
Ángel García-Crespo, Ricardo Colomo-Palacios \\ Juan Miguel Gómez-Berbís, Myriam Mencke \\ Dept. of Computer Science, Universidad Carlos III de Madrid \\ Avenida de la Universidad 30, 28911, Leganés, Madrid, Spain
}

Tel: 0034916245958

E-mail: [angel.garcia, ricardo.colomo, juanmiguel.gomez, myriam.mencke]@uc3m.es

\begin{abstract}
In an increasingly globalized world, changes in organizations have also affected innovation processes. In the Information Technology (IT) domain, corporations are adopting outsourcing and offshoring models for innovation, each time more carefully. The benefits of the combination of both practices, which demonstrate a decrease in corporate costs, indicate an incentive for the adoption of such practices as corporate strategies. In such a scenario, a two-sided challenge presents itself. In the first place, the companies contracting must establish selection measures and strategies for control of their suppliers. Secondly, the business offering outsourcing services must adopt appropriate measures to develop themselves as reliable partners in these types of relationships, a crucial aspect for both parties. The current work presents SemOutQuote, a semantics-based environment for the selection of stable partners for trustworthy innovation processes. The fusion of the capacities of interconnection and communication provided by the internet with the functionalities of the use of semantics represent a novel approach to the management of innovation in organizations.
\end{abstract}

Keywords: Semantics, Semantic Web, Innovation, Outsourcing, Management, Offshoring. 


\section{Introduction}

The generalization of technology has dramatically changed forms of doing business and the associated business models. The capabilities contributed by technology have opened up significant opportunities for emerging world economies. As a result, corporations in developed countries are also noticing these opportunities as a method of improving their own production capacities. In the IT industry, the best production capacities of emerging economies are accompanied by a shortage of qualified professionals. Assessing the situation, taking into account that the end of the first decade of the 21 st century is approaching, in fact it indicates a critical situation, which is particularly evident in the IT field. Examining a study performed for Gartner Inc. by Morello et al. (2007), the lack of talent in the western world is threatening business growth. In this scenario, with the aim of guaranteeing continued business growth of companies, many organizations around the world are performing offshoring practices.

On the other hand, the capacities brought about by technology to support business processes have exhibited a dramatic increase since the growth of the internet. In the field of IT offshoring and outsourcing, the opportunities are intrinsically greater. Taking advantage of this circumstance, the present work proposes a platform for the support of the management of outsourcing service offers for innovation in the IT domain. The offshoring and outsourcing of innovation processes in the IT environment is a very extended practice (Fifarek et al., 2008), which stems from the necessary collaboration of companies to perform technology development (Van de Ven, 2005). According to Munson \& Pelz (1979), innovation can be divided into radical innovation and incremental innovation. Radical innovations are fundamental changes that represent revolution in technology. In contrast, incremental innovations are minor improvements or simple adjustments in existing technologies. The current work focuses on the management of incremental innovation in the IT environment. In order to achieve this, a framework is proposed which enables companies to fully benefit from the capacities of all the participants, and contribute flexibility to the decision process. Given the importance of outsourcing, this work presents a solution for managing innovation in outsourcing processes, which exploits the advantages provided by semantic technologies to represent all of the entities involved in the IT innovation process. The architecture is comprised of a number of components which represent the constituents of this process, including four layers with distinct functions. These include managing the overall software architecture, the parsing of valid business requirements, specification of organizational and personal competencies and constraints, and storage formalisms.

The remainder of the article has been structured as follows. Initially, the basic concepts of outsourcing and offshoring are established and outlined. Secondly, the principal initiatives for the definition and implementation of semantic web tools for the support of business initiatives are discussed. Section 4 details how the SemOutQuote architecture and implementation realize the proposed solution. Lastly, Section 5 presents the principal conclusions of the study, and some suggestions for resolving the problems encountered are outlined, as well as proposals for future research. 


\section{Background: Outsourcing, Offshoring and Innovation.}

Outsourcing describes the use of external resources to execute operational tasks (Grover et al., 1994). Other authors have pointed out that outsourcing must not be seen merely as the firm's acquisition of products and services for the manufacture of goods and provision of services, but as a particular form of strategic alliance (Zineldin \& Bredenlo, 2003). Since the early 1990s, outsourcing has been discussed under diverse aspects in both academic business studies and operational practice (Kakabadse \& Kakabadse, 2000). In the past, the corporate activities which were the subject of outsourcing constituted mechanical activities, or processes with low added value, however, currently many business processes are outsourced (Hoecht \& Trott, 2006). The benefits of outsourcing outlined in the literature are various: Economies of scale (Zineldin \& Bredenlo, 2003), reduction of operational costs (Lacity \& Hirschheim, 1993), access to a skilled workforce (Kakabadse \& Kakabadse, 2002), shorter lead times, increased flexibility and cost efficiency (Nieminen \& Takala, 2006), and the ability to transform fixed costs into variable costs (Alexander \& Young, 1996). On the other hand, there are relevant works of several authors that highlight the risks of outsourcing practices, such as: the hidden costs (Barthelemy, 2001), supplier dependency (Alexander \& Young, 1996), loss of know-how (Doig et al., 2001), and correct supplier selection (Earl, 1996), to cite the most significant ones.

In this scenario, outsourcing represents an opportunity as well as a challenge to many organizations (Barden-Fuller et al., 2005). Leaving aside the necessary balance between the advantages and disadvantages which emerged from the review of the literature, a contribution considered important is that of Smith et al. (2007), who indicate that integration with suppliers constitutes an important managerial skill that must be developed by the entrepreneur.

Regarding offshoring, traditionally offshoring practices involve the movement of low-skilled, labor-intensive manufacturing positions to other countries, especially in the developing world (Doh, 2005). The principal advantages are lower costs, expanded markets and lower prices for consumers (Aron \& Singh, 2005). It is evident that the capacities of offshoring are currently undergoing a paradigm shift due to the increasing abilities provided by IT (Fifarek et al., 2008), research and development in general becoming more knowledge intensive (Lai et al., 2007), in particular, high technology research work in some cases (Fifarek et al., 2008). This is accompanied by the traditional cost difference, due to the shortage of workers in western countries (Morello et al., 2007).

The work proposed here focuses on the management of offshoring outsourcing of innovation, with respect to IT. The focus is on both practices, that is, offshore outsourcing. Offshore outsourcing entails the transfer of parts of a Western firm's value chain activities to partners in emerging economies for a period of time, usually at least a few years (Li et al., 2008). Traditionally, companies pursuing offshore outsourcing migrate peripheral business processes to outsourcing partners in other countries looking for cost advantages (Kakabadse \& Kakabadse, 2005). However, more recent offshore outsourcing initiatives relocate core business process in order to get strategic renewal; this business practice is called 
transformational offshore outsourcing ( $\mathrm{Li}$ et al., 2008). As mentioned above, the IT industry has used offshoring, outsourcing, and offshoring outsourcing since many decades ago. Cases of offshoring and outsourcing of innovation in the IT environment have also been seen (Fifarek et al., 2008), perhaps initiated by the fact that IT innovation is often a result of inter-organizational collaboration (Boland et al., 2006).

Innovation offshoring and outsourcing comes with advantages but also entails disadvantages, it can be seen as a double-edged sword (Gooroochurn \& Hanley, 2007). The advantages have already been discussed, however, the disadvantages are also known, such as opportunism by partner firms, contracting costs, leading to production bottlenecks, and quality issues (Gooroochurn \& Hanley, 2007). In sum, offshoring outsourcing innovation may cause both competitive advantage and disadvantage for partners ( $\mathrm{Li}$ et al., 2008).

Having in mind that the early stage during which consensus building among collaborating firms is critical to establish favorable conditions for the innovation (Andersson et al., 2008), this paper proposes a novel and promising architecture designed to manage the early stages of offshoring outsourcing: the selection of appropriate and competent partners. In particular, semantics are used to enhance partner selection based on workers and firm competency.

\section{Using Semantic Technologies to Innovate the Offshore Outsourcing}

Traditional innovation focuses on knowledge management optimization techniques in order to improve scenarios where knowledge is distorted, suppressed or misappropriated due to organizational or personal motives (Alter, 2006).

This section details the use of semantic technologies to innovate the process of offshore outsourcing. The forthcoming of semantic technologies gathering significance in current developments offers new possibilities towards interweaving those distributed, heterogeneous and changing sources into added value infrastructures. Semantic technologies offer a model approach where the underlying data and processes can be interchanged declaratively, increasing flexibility and decreasing load on the IT organization. For example, in Gómez-Berbís et al. (2008), ProLink, a semantics-based social network, harnesses the possibilities of reusing, gaining knowledge, expertise and lessons learned from previous software projects. Since initial research efforts in the field, semantic technologies have gathered a significant amount of research and development initiatives which have in common the focus on extending current web technology with machine-understandable metadata, aimed at providing layered services. In another example, Colomo-Palacios et al. (2008) show how semantic technologies and social web can be used to enhance collaborations in software development projects.

The reasons for using semantic technologies are twofold:

- Formal Protocol Description: Nowostawski et al. (2001) envisage interaction protocols as a template of sequences of expected communicative acts or speech acts organized into roles. Speech acts are a conceptual model whose aim is to represent pure communication acts. This model is included here for its importance regarding communication theory and the technologies based on the speech act model. The 
speech act theory was developed to systematically classify types of these acts regarding their intention or attitude when expressed. A speech act is, in fact, the performance of several acts at once, distinguished by different aspects of the speaker intention (Austin, 1962). This interaction protocol is compatible with the definition of protocol specified by the Foundation for Intelligent Physical Agents (FIPA): " $a$ common pattern of dialogues used to perform some generally useful task. The protocol is used to facilitate a simplification of computational machinery needed to support a given dialogue task between agents" (FIPA, 1997). By using semantic technologies, a formal description of the protocol can be realized, which can be used by the stakeholders of the offshore outsourcing process.

- Using Logics for Knowledge Representation: Logics encompasses the study of reasoning principles. Therefore, logic builds formal languages for expressing knowledge, semantics and automatic reasoning engines to deduce (infer) conclusions. Logics are one of the main mechanisms of knowledge representation, which has been applied in artificial intelligence in general and the World Wide Web in particular. Logics offer high level languages to express knowledge and also have high expressive power. Also, knowledge representation has a well defined formal semantics for assigning unambiguous meaning to logical declarations.

Particularly, the focus of the current work in the sections which follow may be divided into using a semantic description of the data involved in the offshore outsourcing process, and developing a protocol for the interaction. In the latter, a protocol identifies roles and sequences of communicative acts and specifies relations between roles. A role is just an identifier of a single sequence of acts executed by a single entity. The second layer is the conversation layer. A conversation is a particular instance of a protocol or set of protocols; an ongoing sequence of messages exchanged between two or more agents. The protocol is just a template or pattern, whereas a conversation is an instance of a given set of templates. This definition is also compliant with the definition of the FIPA: an ongoing sequence of communicative acts exchanged between agents relating to some ongoing topic of discourse (FIPA, 1997). Finally, the Policy layer contains policies, a policy being a collection of rules and interaction specifications that guide a particular path in a conversation space. An example of this approach is to define two participants as buyer and seller. The buyer can only have three actions (ask, accept, reject) and the seller two (propose, sell). This defines the protocol. The participants follow this protocol to make the actual conversation, that is, ask-propose-accept-sell.

In the next section, the SemOutQuote architecture is outlined, which is based on a fully-fledged set of interlinked software components.

\section{SemOutQuote}

The proposed SemOutQuote architecture is based on a number of software components, which will be detailed in the following subsections. Process Algebras have been selected as the formalism to describe the semantics of the protocol. The term process algebra is used in different contexts, but usually, it refers to a formalism based on an algebra used to model the 
behavior of a system. More concrete examples of modeled behaviors with process algebras could be the execution of a software system, or the actions of a machine.

\subsection{Formal Protocol Representation}

The Pi calculus (Sangiorgi, 2004) is a mathematical model of processes whose interconnections change as they interact. It is mainly based on the CCS (Calculus for Communicating Systems) theory developed by Milner (1989), (which is an algebraic calculi model for the analysis of concurrent systems), but adds mobility as a new feature. This feature, mobility, implies that a communication link between two processes can be transferred from one to another. This makes the calculus very adequate for modeling systems where the resource access varies over time.

One of the cornerstones ideas of the Pi calculus is that the links belong to the same category as the transferred objects. This is not only a simple variant of value-passing process algebra, but also it defines a way to provide a scope for the transferable objects. For instance, if two processes are sharing the same link and one sends this link to a third, then a link exists which is accessed by three processes, but distinct to any other link accessed by any other process. The basic definitions are similar to those in CCS, although the syntax varies. Firstly a set of names are assumed, which function as communication ports, variables and data values, and a set of identifiers which represent processes. These are outlined as follows:

- The empty process 0 , which cannot perform any action.

- An output prefix $\overline{\mathrm{a}}(\mathrm{x}) \cdot \mathrm{P}$ The intuition is that a name is received along a name. So $\bar{a}$ can be thought as an output port and $x$ as a datum sent out from that port.

- An Input prefix a(x) $\mathrm{P}$ meaning that a name is received along a name $a$, and $x$ is a placeholder for the received name. This may be considered as an input port, and $x$ as a variable which will get its value for the input along $a$.

- A silent prefix, $P$, which represents a process that can evolve to $P$ without interaction with the environment.

- A sum $P+Q$ representing a process that can act either $P$ or $Q$

- A parallel composition $P \mid Q$, which represents the combined behavior of $P A\left(y_{1}, \ldots, y_{n}\right)$ and $Q$ executing in parallel. The components $P$ and $Q$ act independently and may also communicate if one performs an output and the other an input along the same port. $A\left(y_{1}, \ldots, y_{n}\right)$ represents how a defined agent will be instantiated. 
- A match if $x=y$ then $P$. The process will behave as $P$, if and only if $x$ and $y$ are the same.

- A mismatch if $x \neq y$ then $P$. The process will behave as $P$, if and only if $x$ and $y$ are not the same.

- A restriction $(v x) P$. The process behaves as $P$ but the name $x$ is local, meaning it can also be used as a communication port between $P$ and its environment.

- An identifier $A\left(y_{1}, \ldots, y_{n}\right)$ where $n$ is the arity of $A$.

The match and mismatch constructs are the only possible tests that can be performed in the Pi calculus: the objects transmitted are just names and these have no structure and no operators, so it is only possible to compare them for equality.

A simplified version of the current example is based on the ContractNet protocol (Smith, 1980). The protocol is briefly explained as follows. A customer sends an RFQ (Request for Quotation) message to the supplier. This message is a request to the supplier asking for the provision of product information, with regard to product availability, shipping, and price. The supplier replies with a quote. The customer sends a message for ordering goods to the supplier. The supplier confirms the order. Firstly, this example is the result of combining two processes that are being executed in parallel, $P$ in the sender and $Q$ in the receiver. So the whole interaction, a process $\mathbf{R}$ could be described as $R=P \| Q$. $\|$ represents a parallel composition.

To describe $P$, the $P^{1}, P^{2}, P^{3}$ and $P^{4}$ processes are defined. However, in the Pi calculus it is essential to distinguish between input prefixing and output prefixing. Two output prefixes are defined: $\overline{r f q}(x) . P$ and $\overline{\operatorname{order}}(x) . P$. The definition of two input prefixes is also required: quote $(x) . P$ and confirm $(x) . P$. The $P$ process is described as follows:

$$
\begin{aligned}
& P=\overline{r f q(x)} \cdot P^{1} \\
& P^{1}=\operatorname{quote}(x) \cdot P^{2} \\
& P^{2}=\overline{\operatorname{order}}(x) \cdot P^{3}
\end{aligned}
$$




\section{Macrothink

$P^{3}=\operatorname{confirm}(x) \cdot P^{4}$

To describe $Q$, the $Q^{1}, Q^{2}, Q^{3}$ and $Q^{4}$ processes are defined. Again, in Pi calculus the distinction between input and output prefixing is made. Two input prefixes are defined: $r f q(x) \cdot Q$ and $\operatorname{order}(x) \cdot Q$. The parameter $(x)$ is a variable (name) that can be also

sent. Two output prefixes are also defined: $\overline{q u o t e}(x) \cdot Q$ and $\overline{\operatorname{confirm}}(x) \cdot Q$. The $Q$ process is described as follows:

$\mathrm{Q}=r f q(x) \cdot Q^{1}$

$Q^{1}=\overline{q u o t e}(x) \cdot Q^{2}$

$Q^{2}=\operatorname{order}(x) \cdot Q^{3}$

$\mathrm{Q}^{3}=\overline{\operatorname{confirm}}(x) \cdot Q^{4}$

In the next section, the software architecture is described, having into account that the formal representation of the protocol is modeled with the Pi calculus semantics described in this section.

\subsection{Architecture.}

The SemOutQuote architecture presents a set of significant decisions about the organization of the software system, by selecting a number of structural elements and their interfaces. Components might be related to the behavior as specified in the collaboration among those elements, turning those structural and behavioral elements into progressively larger subsystems and the architectural style that guides this organization.

In this section, a loose-coupled view of the architecture is introduced. This view is provided with a layered design. The advantage of layering is the conceptual distinction and functionality between layers. Each layer has a particular and precise functionality. Layers observe a dependency relationship from down to up. This means that the upper layers rely on some functionality of the lower layers. Layered architectures are used in different domains but particularly in communication systems where each layer implements a different aspect of the information exchange. The layer view is shown in Figure 1. 


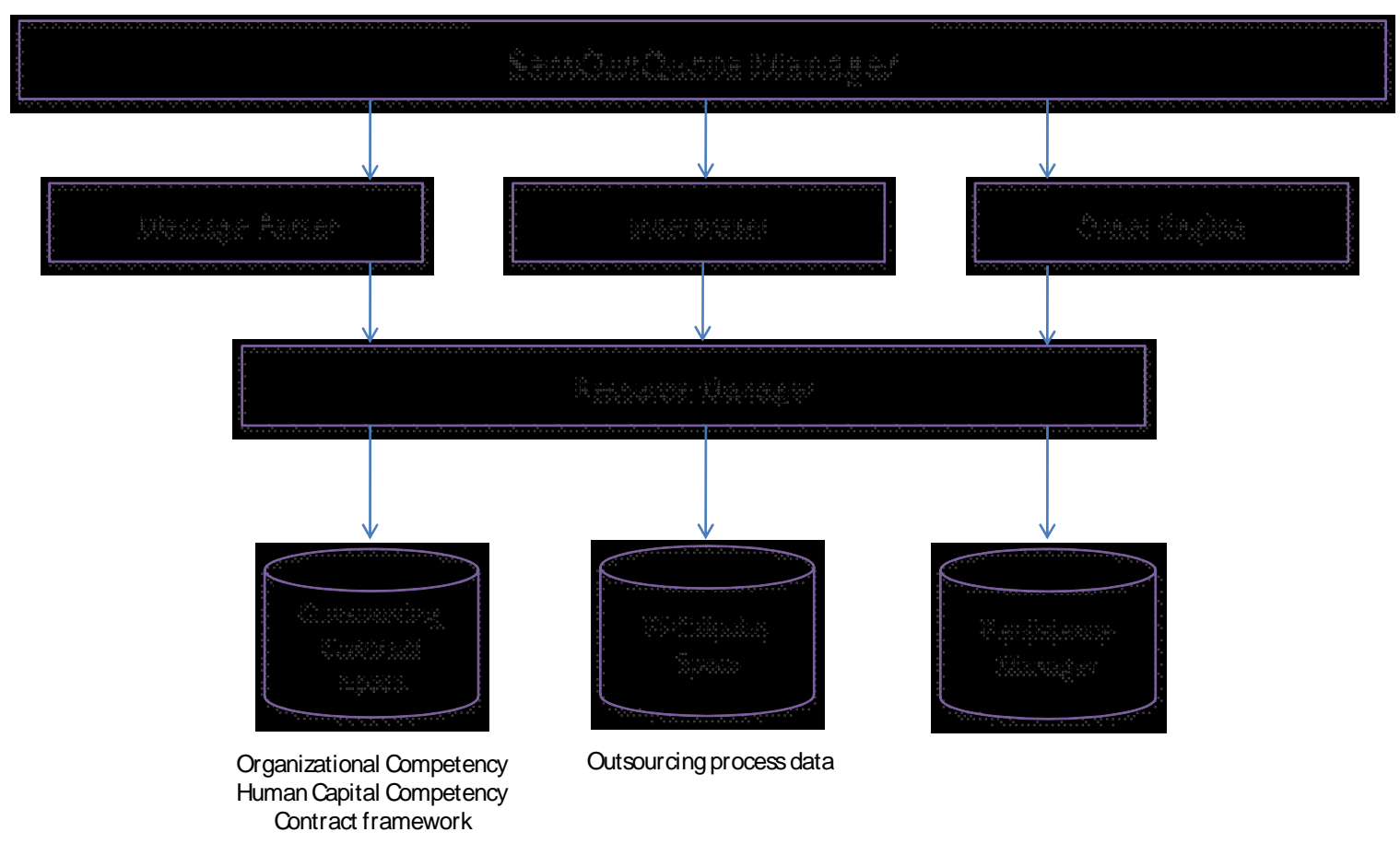

Figure 1. SemOutQuote Architecture.

First of all, the upper layer is composed of the SemOutQuote manager, which organizes and coordinates the overall software architecture functionality. The next layer consists of the following elements:

- The lexer or lexical analyzer reads the contract, process and Pi calculus specifications and sends them to the parser.

- The parser recognizes valid and sound expression by analyzing the syntax structure of the set of tokens passed to it from the Interpreter.

- The Interpreter reads one statement at a time, those statements being part of the Pi calculus specification language described in the previous section. It translates each statement to machine language and executes the machine language statement, then continues with the next statement.

The third layer is managed by the resource manager, which handles the functionality of the following three components:

- Outsourcing contracts specifications: fundamentally, the outsourcing contracts specifications encompass three different types of concepts. These concepts are data-oriented and they refer to the following:

o Organizational competencies: both entities involved in the outsourcing process have different experience in terms of the above mentioned criteria to make the contract come true, such as portfolio or previous similar assignments.

o Non-functional constraints: deadlines, price or time are also taken into 
account and described semantically.

o Personal competencies: both entities in the outsourcing process are matched from "personal" skills and similarity.

- Pi calculus specs: Pi calculus was selected as the formalism to describe the semantics of the protocol. In this case, the Pi calculus models the interaction protocol among the different mechanisms.

- Persistence manager: this extra component ensures the persistence of the different elements or objects being used as a back-up mechanism by the resource manager.

In this section, the SemOutQuote architecture has been described. In the next section, a use scenario will be outlined which demonstrates the functionality of the architecture.

\subsection{Use Scenario}

Finally, the use of SemOutQuote with a real-world case study scenario will be illustrated, which demonstrates the breakthroughs of the proposed system. Taking into account publication space limitations and the intrinsic complexity of outsourcing, the outsourcing process will be illustrated with a detailed example. SemOutQuote is installed in an accessible server, and acts as a market place for offers of outsourcing services and for companies requiring outsourcing services. A number of different people are involved in the process, both in the company who requires outsourcing and the company providing the outsourcing. These people are likely to be the end users of the system, and include project managers, vice presidents, innovation management, and all other entities involved in the innovation process. Suppose that the company Enterprise Inc., dedicated to software development, wishes to externalize part of its innovation process. The said company requires the incorporation of a web service in the new release of its key product, the TOOL system for hotel client management.

The particular objective of the web service is to be responsible for distributing specific weather forecast information to clients, with the aim of being used for automatic programming of leisure and tourist activities which respond to the preferences of hotel clients and climatic conditions.

The web service should be programmed, compatible with the rest of the TOOL program, using open Java code standards. For the development of the project, the company expects a time span of 4 months from the publication of the demand for the service. Additionally, the company should perform maintenance throughout the useful life of the tool. The process is considered by Enterprise Inc. as an innovation process, given that the technology they wish to develop is not available on the market, and is considered a challenge which Enterprise Inc.'s resources are not able to fulfill in its entirety. The usual practice in the countries where Enterprise Inc. sells TOOL licenses requires a service which distributes the forecast using SOAP servers. The complexity and the necessary development includes, apart from the complete development of the service, the automatic interpretation of the data, and the georeferencing of this data from the viewpoint of the TOOL application. 
Considering its requirements, Enterprise Inc. introduces three types of information into the system. The first type of data concerns the competences and experience of the people comprising the development team. These companies will be classified using the pertinent ontology, and divided into technical and functional competencies. Regarding technical competencies, and data input will relate to the development of a web service using Java technology, and from the functional perspective, it will include elements related to meteorology and hotel services. Secondly, data will be introduced relating to organizational competencies. In this case, elements will be coded which represent the desired experience, in outsourcing innovation processes in technology, and more specifically, software development. Lastly, administrative data will be introduced, such as deadlines, contract forms, payments, etc.

From the point of view of the offer, the Chinese company Ri Lao, located in Beijing, China, has been providing services related to the development of software for Geographical Information Systems (GIS) since 2000. It holds experience in the analysis of meteorological data in the recent Beijing Olympics 2008, in particular, establishing personalized forecasting services for different athletic events, the triathlon and pentathlon. It does not have experience in outsourcing, has qualified professionals, and is prepared to open to this market, which it considers fundamental from the point of view of its strategic evolution.

Furthermore, the Indian company Aiyam dedicates itself specifically to the outsourcing of development environments of commercial software, whether it is innovative or simply driven by market requirements. It has a spectrum of qualified software engineers, experts in the development of software using Java, and for open code software. It is evident that it does not have functional experience in the management of weather forecast data, even though it manages XML information and has experience with GIS systems.

Taking into account the complete scenario, SemOutQuote will analyze the demand and the base of service providers, displaying to Enterprise Inc. two valid candidates, offering two recommendations, one of which includes a major valuation of the organizational competency, the Indian company Aiyam, with experience in outsourcing and offshoring processes. Furthermore, Aiyam displays less alignment in aspects of individual functional competency, not having performed tasks relating to forecasts. Continuing the analysis, the Chinese company $\mathrm{Ri}$ Lao is positively valued in functional experience, but valued lower in organizational competency, not holding experience in offshore outsourcing. The data, jointly with economic factors, which have been omitted in this example, will be presented to the management of Enterprise Inc. This corporation will make their decision based on the recommendations, as well as their own interests. Determination of the decision process is not desired, rather, the objective is to equip the decision makers with a new support tool for the decision process.

Once the responsibles have made their decision, the project is realized, introducing elements which build experience and extend the portfolio of projects of both companies, with the objective that future sales are amplified with information relative to the process. The typology of the information will include, from the service provider side: fulfillment of 
deadlines, quality of the process, quality of the product, innovation perceived, and level of satisfaction with the work team. From the side of the contractor, information will be coded relative to the reliability of the specifications, support for the innovation process, and fulfillment of wage payments.

\section{Conclusions and future work.}

The current research work has presented a tool which aims to serve as a meeting point between the supply and demand of innovation projects in the IT field. Compared with previous initiatives, the principal novelty of the present work is the use of the semantic web and its associated semantic technology in the design and use of the SemOutQuote tool. The capacities of the semantic web enable the market place for the contracting of services to evolve, and contribute functionalities unexploited until now. The use of ontologies in this type of environment creates an advantage which stems from the ability to classify knowledge, which these types of methods intrinsically possess.

Apart from the enormous search and specific criteria matching capabilities which the use of semantics provides, accurate input is provided to organizational systems, which guarantees the precision of the data. In this way, creation of inconsistencies is avoided and thus eliminated. Lastly, the relevance of the practice of outsourcing and offshoring IT innovation processes defines a very fertile research field, both from the point of view of organizations and business processes, as well as the technologies performing such processes. Following this line of thinking, and bearing in mind the focus adapted by SemOutQuote, a future extension of the tool is proposed to carry out a monitoring of the management of the innovation process, which includes a modeling of business processes, focusing on follow-up and thereby enabling supervision of the work by third parties.

\section{References}

Alexander, M. and Young, D. (1996). Outsourcing: where's the value?. Long Range Planning, 29 (5), pp. 728-730.

Alter, S. (2006). Goals and Tactics on the Dark Side of Knowledge Management. Proceedings of the $39^{\text {th }}$ Hawaii International Conference on System Sciences (HICSS '06), 7 (January 4-7, 2006), p. 144.

Andersson, M., Lindgren, R. and Henfridsson, O. (2008). Architectural knowledge in inter-organizational IT innovation. Journal of Strategic Information Systems, 17 (1), pp. 19-38.

Aron, R., and Singh, J. (2005). Getting offshoring right. Harvard Business Review, 83 (12), pp. 135-143.

Austin, J. (1962). How to do things with words. Cambridge, Mass.: Harvard University Press.

Barden-Fuller, C., Targett, D. and Hunt, B. (2005). Outsourcing to outmanoeuvre: outsourcing re-defines competitive strategy and structure. European Management Journal, 18 (3), pp. 285-295. 
Barthelemy, J. (2001). The hidden costs of IT outsourcing. Sloan Management Review 42 (3), pp. 60-69.

Boland, R.J., Lyytinen, K. and Yoo, Y. (2007). Wakes of innovation in project networks: the case of digital 3-D representations in architecture, engineering, and construction. Organization Science 18 (4), pp. 631-647.

Colomo-Palacios, R., Gómez-Berbís, J.M., García Crespo, A. and Puebla-Sánchez, I. (2008). Social Global Repository: using semantics and social web in software projects. International Journal of Knowledge and Learning, 4 (5), pp. 452-464.

Doh, J. (2005). Offshore outsourcing: implications international business and strategic management theory and practice. Journal of Management Studies, 42 (3), pp. 695-704.

Doig, S.J., Ritter, R.C., Speckhals, K., and Woolson, D. (2001). Has outsourcing gone too far?. McKinsey Quarterly, 4, pp. 24-37.

Earl, M. (1996). The risks of outsourcing IT. Sloan Management Review, 37 (3), pp. 26-32.

Fifarek, B.J, Veloso, F.M. and Davidson, C.I. (2008). Offshoring technology innovation: a case study of rare-earth technology. Journal of Operations Management, 26 (2), pp. 135-140.

FIPA. Foundation for Intelligent Physical Agents (1997). Agent Communication Language (ACL) Geneva, Switzerland. [Online] Available: http://www.fipa.org/. (December 18, 2008).

Gooroochurn, N. and Hanley, A. (2007). A tale of two literatures: transaction costs and property rights in innovation outsourcing. Research Policy, 36 (10), pp. 1483-1495.

Gómez-Berbís, J. M. Colomo-Palacios, R. García-Crespo, A. and Ruiz-Mezcua, B. (2008). ProLink: a semantics-based social network for software project. International Journal of Information Technology and Management, 7 (4), pp. 392-404.

Grover, V., Cheon, M.J. and Teng, J.T.C. (1994). A descriptive study on the outsourcing of information systems functions. Information \& Management, 27 (1), pp. 33-44.

Hoecht, A. and Trott, P. (2006). Innovation risks of strategic outsourcing. Technovation, 26 (5/6), pp. 672-681.

Kakabadse, A. and Kakabadse, N. (2002). Trends in outsourcing. European Management Journal, 20 (2), pp. 189-198.

Kakabadse, A. and Kakabadse, N. (2005). Outsourcing: current and future trends. Thunderbird International Business Review, 47 (2), pp. 183-204.

Kakabadse, N. and Kakabadse, N. (2000). Outsourcing: a paradigm shift. Journal of Management Development, 19 (8), pp. 670-728.

Lacity, M. and Hirschheim, R. (1993). Information systems outsourcing: myths, metaphors and realities. Chichester: Wiley.

Lai, E.L.C., Riezman, R. and Wang, P. (2007). Outsourcing of innovation. Economic Theory. 


\section{Macrothink \\ International Journal of Management Innovation Systems \\ ISSN 1943-1384 \\ 2009, Vol. 1, No. 1: E3}

In Press.

Li, Y., Liu, Y., Li, M. and Wu, H. (2008). Transformational offshore outsourcing: empirical evidence from alliances in China. Journal of Operations Management, 26 (2), pp. 257-274.

Milner, R. (1989). Communication and Concurrency. Englewood Cliffs: Prentice-Hall International.

Morello, D., Kyte, A. and Gomolski, B. (2007). The Quest for Talent: you ain't seen nothing yet. Gartner Inc. [Online] Available: http://www.gartner.com/DisplayDocument?ref=g_search\&id=569115\&subref=advsearch. (December 17, 2008).

Munson, F.C. and Pelz, D. C. (1979). The innovating process: a conceptual framework. Working Paper, University of Michigan.

Nieminen, P. and Takala, J. (2006). Achieving better on-time delivery performance with the help of internal dependencies in the production. International Journal of Management and Enterprise Development, 3 (1/2), pp. 181-190.

Nowostawski, M., Purvis, M., and Cranefiedl, S. (2001). A layered approach for modeling agent conversations. In Proceedings of the 2nd International Workshop on Infrastructure for Agents, MAS, and Scalable MAS, 5th International Conference on Autonomous Agents, Montreal, pp. 163-170.

Sangiorgi, D. and Walker, D. (2004). The Pi-calculus: A theory of mobile processes. New York, NY: Cambridge University Press.

Smith, R.G. (1980). The contract net protocol: high-level communication and control in a distributed problem solver. IEEE Transactions on Computers, 29(12), pp. 1104-1113.

Smith, W.L., Schallenkamp, K. and Eichholz, D.E. (2007). Entrepreneurial skills assessment: an exploratory study. International Journal of Management and Enterprise Development, 4(2), pp. 179-201.

Van de Ven, A.H. (2005). Running in packs to develop knowledge-intensive technologies. MIS Quarterly, 29(2), pp. 365-378.

Zineldin, M. and Bredenlow, T. (2003). Strategic alliance: synergies and challenges. A case of strategic outsourcing relationship 'Sour'. International Journal of Physical Distribution \& Logistic Management, 33(5), pp. 449-464. 